\title{
Analysis and Research on Guiding Cone Angle Parameter of Spray Fan-Pipe
}

\author{
Zhang Fajun", Zhang Feng, Yang Xianwei, Yang Jingjign, Li Linzi \\ College of Mechanical \& Power Engineering of China Three Gorges University, Yichang, China \\ Email address: \\ doctorzyy@126.com (Zhang Fajun), 1425808925@qq.com (Zhang Feng), 542481248@qq.com (Yang Xianwei), \\ 447445604@qq.com (Yang Jingjign),653549221@qq.com (Li Linzi) \\ ${ }^{*}$ Corresponding author
}

\section{To cite this article:}

Zhang Fajun, Zhang Feng, Yang Xianwei, Yang Jingjign, Li Linzi. Analysis and Research on Guiding Cone Angle Parameter of Spray Fan-Pipe. International Journal of Fluid Mechanics \& Thermal Sciences. Vol. 2, No. 3, 2016, pp. 16-21. doi: 10.11648/j.ijfmts.20160203.11

Received: September 28, 2016; Accepted: October 29, 2016; Published: November 30, 2016

\begin{abstract}
Guiding cone angle is an important parameter in the design of the spray fan, the difference of guiding cone angle has a significant impact on the spray effect and service life of the fan. Based on this, the numerical simulate of fluid-solid coupling for the pan is carried out by using the RNG k- $\varepsilon$ model, turbulence equations and SIMPLEC algorithm, then analyze the fan's changes of the flow field and structural under the different cone angle. Simulation results show that with the increase of the cone angle, the pressure and speed of the flow field, the stress and strain of the blade also increased, but when it over a certain angle, the result will decreases. Further analysis shows when the guiding cone angle of the fan is $20^{\circ}$, the performance of the fan is optimal. The results of this analysis can provide a theoretical basis for the design of the fan.
\end{abstract}

Keywords: Spray Fan, Fluid-solid Coupling, Guiding Cone

\section{Introduction}

There are two major kinds of spray fan researching in china. The first is the orchard air assisted spray fan which is the axial inlet air and both sides outlet air: Yang Chun [1] et al through numerical simulation and experiment, found the fan with the current collector and rectifying cap raised the full pressure and efficiency of the fan by $5.2 \%$, after analysis of the two cases of the motor and the hub at different radius and whether a current collector or a rectifier cap was added to the import. Lv Xiaolan [2] et al studied the effects of spray fan's parameters to the droplet deposition; Guo Feng [3] et al made three dimensional modeling and Simulation of spray fan whose entrance is cylindrical by using numerical simulation technology. Another kind is the turret air assisted spray fan: Song Shuran [4] et al optimized the design of the spray tube structure of the air spray fan by using numerical methods and orthogonal test, the factors that influence the pressure loss and the air outlet velocity of the spray-duct are obtained in turn: outlet diameter 、 length of cylinder spray-duct、 length of conical spray-duct; Liu Qing [5] et al made the spray effect increased by $22 \%$ to $46 \%$ by installing a guiding vane in the spray-duct outlet of the
9WZCD-25 air assisted spray fan.

The computer modeling of the spray fan and its working environment parameters are carried out abroad, and the spray process is simulated. The spray effect has great advantages on the efficiency and the economy, but most of them are the axial inlet air and both sides outlet air. Delele [6] et al designed a computational fluid dynamics model to study the spray effect of a spray fan in the flow field generated by the axial flow fan; Endalew [7] et al established the three-dimensional model of the two fan mixed flow orchard air assisted spray fan by using CFD technology, the distribution of the spray flow velocity in different canopy density regions is predicted by Using this model, Some foreign scholars also studied the droplets' penetration and performance improvement of spray fan $[8,9]$.

Cone angle is one of the most important parameters in the design of spray fan, the designs of the cone angle size are not uniform currently and no feasible theory can be used as a basis for design. Although its variation range is small, the change value has an important influence on the spray effect, because after atomization and separation by highly speed rotating of impeller blade, the liquid in spray-duct need to be discharged through the cone, and the size of the cone angle affect the internal pressure, especially in the early stage of work, maybe 
causing severe overshoot phenomenon and then damage the blade and the whole structure of the fan. Therefore, the study of the parameters of the nozzle cone angle has important engineering significance for the design of the cone angle.

In this paper, the model is numerically analyzed by using the constant coupling method which is based on the vehicle mounted sprayer. In order to get a better angle of guiding cone angle and provide the theory reference for the design of the vehicle mounted sprayer, analysis on pressure of spray fan, velocity, stress and strain of model under different guide cone angle are carried out.

\section{Mathematical Simulation}

\subsection{Geometric Model and Mesh Division}

The three-dimensional model of the fan is established by using SolidWorks. Using ADINA analyze the model of spray fan. The motor rated power is $5.5 \mathrm{KW}$, the rated speed is $1430 \mathrm{rpm}$. The diameter of the rotary shaft is 0.08 meters. The related parameters of the blade model of the fan: the outer diameter of the fan is 0.4 meters; the diameter of shaft that the blades connected is 0.08 meters; the shaft length of the rotary shaft is 0.08 meters; the blade length, width and thickness of the study model are 0.16 meters, 0.06 meters and 0.01 meters; the number of blades is 3 , and the blade is 120 degrees equiangular distribution; the blade surface is a quasi streamline surface, and installation angle is 35 degrees.

Using FCBI-C unit, the degree of freedom of the element is defined at the center of the unit which conform to the fluid-solid coupling problem. Because of the solution of the spray fan fluid-solid coupling analysis is mainly concentrated in the two intersecting interface, the calculation of the coupling part needs the mesh quality to be very high. In order to obtain high quality mesh, we must adopt the method of dividing the grid into the block which can reduce the number of the grid the fullest extent, and does not affect the accuracy of the solution. For blade sections, because of their regular geometrical shape and the small gradient change of flow field of various physical quantities, the mapping grid adopt (rule based) 8 node hexahedron grid division. For the rotary shaft, because of the complexity of the geometric structure, dividing mapping mesh exists certain difficulties, in addition, the changes of some physical quantity in the flow field are relatively big, so the free mesh adopts (free-form) 8 node hexahedron grid division, and encrypt the area where the stress concentrate in the blade root. Fig. 1 and Fig. 2 respectively are the grid partition of the blade and the flow field.

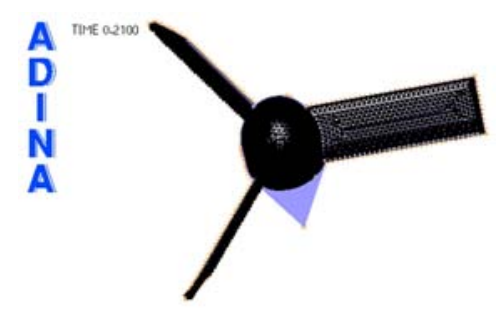

Fig. 1. Axial fan structured grid.

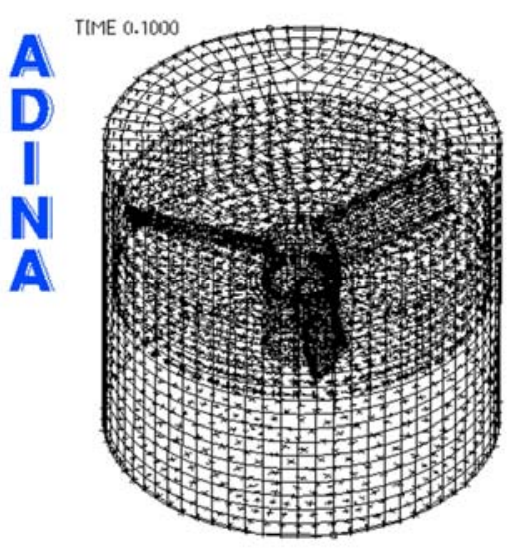

Fig. 2. Axial fan flow field grid.

In order to accurately reflect the influence of the overshoot phenomena on the spray-duct flow field and the blades, the first 20 steps' coupling calculation step time is taken as $0.005 \mathrm{~s}$, and total is $0.1 \mathrm{~s}$; the afterwards 200 steps coupling calculation step time is taken as $0.01 \mathrm{~s}$, and total is $2 \mathrm{~s}$. This selection of step time can characterize some motion characteristics of the fan in the running process, and guarantee the accuracy of the results.

\subsection{Mathematical Model}

The flow field in the spray fan is simulated, and considering the strong rotating flow field has a strong anisotropy, the Reynolds stress model started from turbulence anisotropy is used to simulate the gas phase flow field, and control equations are as follows:

Continuity equation:

$$
\frac{\partial v_{i}}{\partial x_{i}}=0
$$

Momentum equation:

$$
\begin{aligned}
\rho v_{j} \frac{\partial v_{j}}{\partial x_{j}}= & -\frac{\partial p}{\partial x_{i}}+\frac{\partial}{\partial x_{j}}\left[\mu\left(\frac{\partial v_{i}}{\partial x_{j}}+\frac{\partial v_{j}}{\partial x_{i}}\right)\right] \\
& +\rho g_{i}-\frac{\partial}{\partial x_{j}}\left(\rho \overline{v_{i} v_{j}}\right)
\end{aligned}
$$

The Reynolds stress which stands for turbulent pulsating effect is solved by using Reynolds stress transport equation:

$$
\begin{aligned}
& \frac{\partial}{\partial x_{k}} \rho v_{k} \overline{v_{i} v_{j}}=P_{i j}+\phi_{i j}+\frac{\partial}{\partial x_{k}} \\
& {\left[\left(\mu+\frac{\mu_{t}}{\sigma_{k}}\right) \frac{\partial}{\partial x_{k}}\left(\overline{v_{i} v_{j}}\right)\right]-\frac{2}{3} \rho \varepsilon \delta_{i j}}
\end{aligned}
$$

Where, stress generating item: 


$$
P_{i j}=-\rho\left[\overline{v_{i} v_{k}}\left(\partial v_{j} / \partial x_{k}\right)+\overline{v_{j} v_{k}}\left(\partial v_{i} / \partial x_{k}\right)\right]
$$

Stress-strain terms:

$$
\begin{aligned}
& \phi_{i j}=-C_{1} \rho(\varepsilon / k)\left[\overline{v_{i} v_{j}}-(2 / 3) k \delta_{i j}\right]- \\
& C_{2}\left[P_{i j}-(1 / 3) P_{k k} \delta_{i j}\right]
\end{aligned}
$$

The turbulent dissipation ratesis solved by solving transport equations:

$$
\begin{aligned}
& \frac{\partial}{\partial x_{i}}\left(\rho v_{i} \varepsilon\right)=\frac{\partial}{\partial x_{j}}\left[\left(\mu+\frac{\mu_{t}}{\sigma_{\varepsilon}}\right) \frac{\partial \varepsilon}{\partial x_{j}}\right] \\
& +\frac{\varepsilon}{k}\left(\frac{1}{2} C_{\varepsilon 1} p_{i i}-C_{\varepsilon 2} \rho \varepsilon\right)
\end{aligned}
$$

where, $\mathrm{C} 1=1.8, \mathrm{C} 2=0.6, C_{\varepsilon 1}=1.44, C_{\varepsilon 2}=1.92, \sigma_{k}=1, \sigma_{\varepsilon}$ $=1.3$. PRESTO method is used to deal with the pressure gradient, and the convective terms of each equations are discretized by QUICK difference scheme. The coupling of pressure and velocity is calculated by SIMPLE-C method.

\section{Results and Analysis}

\subsection{Flow Field Pressure and Velocity Analysis}

1) The influence of cone angle on flow field pressure

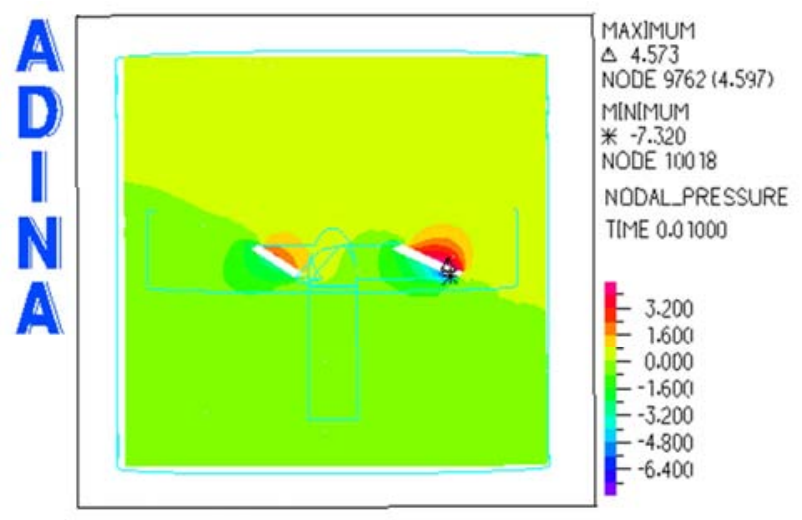

(a) The pressure of section $\mathrm{X}$ under $0^{\circ}$

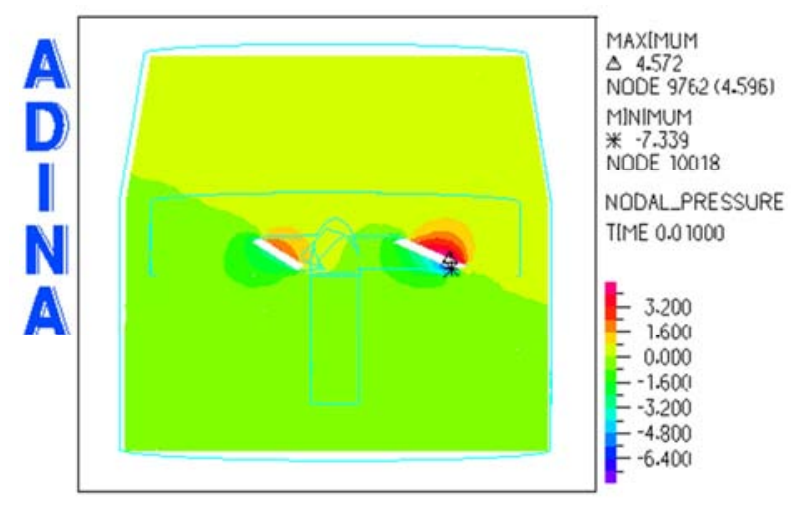

(b) The pressure of section $\mathrm{X}$ under $10^{\circ}$

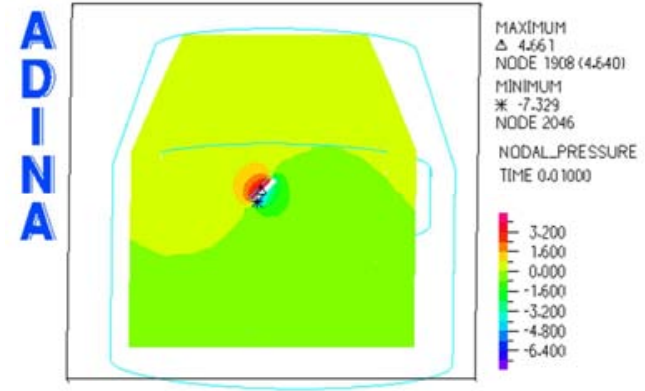

(c) The pressure of section $\mathrm{X}$ under $20^{\circ}$

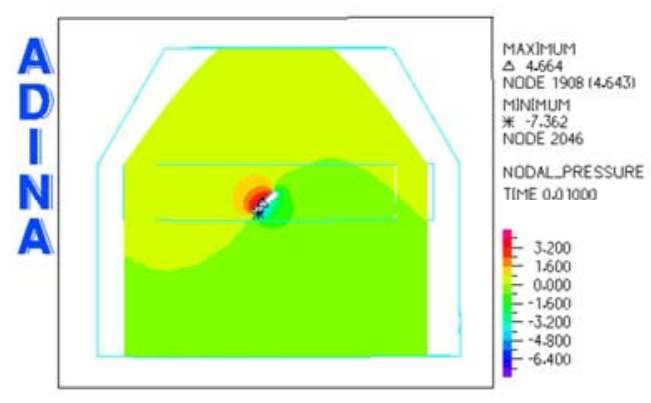

(d) The pressure of section $\mathrm{X}$ under $30^{\circ}$

Fig. 3. The pressure of flow field under different cone angle.

Figure 3 is the pressure distribution chart of spray fan on $\mathrm{x}=0$ longitudinal section which take the maximum node as a basic point under different guiding cone angle. From Figure 5 (a) and (b), it can be clearly seen that the flow field has an axial symmetry and distinct separation happened on which the cone angle changes, and the upper surface pressure of blade is larger at this point. When the fan guide cone angle is 20 degrees to 30 degrees, the pressure range have increasing trend. Table 1 shows the pressure change to have more detailed observation of the changing trend.

From table 1, when the guide cone angle increases, the maximum flow field pressure in the spray-duct increases at the same time, and the absolute value of the minimum pressure increases. However, when the cone angle is 20 degrees, the spray-duct pressure increases to a certain value, for the meantime the negative pressure also reduced to a certain value. The greater pressure can ensure the energy required for transporting fog particles, and the smaller differential pressure will ensure the amplitude value of alternating load decreases in the operation, and service life of the spray fan can be extended.

Table 1. The pressure of $X$ section and $Z$ section under different cone angle.

\begin{tabular}{lllll}
\hline \multirow{2}{*}{ cone angle } & \multicolumn{2}{l}{ Pressure of $X$ section $(P a)$} & \multicolumn{2}{l}{ Pressure of $Z$ section (Pa) } \\
\cline { 2 - 5 } & MAX & MIN & MAX & MIN \\
\hline $0^{\circ}$ & 4.573 & -7.32 & 1.865 & -7.32 \\
$10^{\circ}$ & 4.572 & -7.339 & 1.851 & -7.339 \\
$20^{\circ}$ & 4.661 & -7.329 & 1.867 & -7.329 \\
$30^{\circ}$ & 4.664 & -7.362 & 1.86 & -7.362 \\
\hline
\end{tabular}

2) The influence of cone angle on flow field velocity

In order to ensure the effective transmission of fog particles, in addition to the flow field pressure, velocity distribution of 
flow field which is parallel to the spray-duct axis direction (that is $\mathrm{Z}$ axis direction) must also be considered. So this article did a comparative analysis on the speed of the spray-duct flow field in the direction of $\mathrm{Z}$ axis.

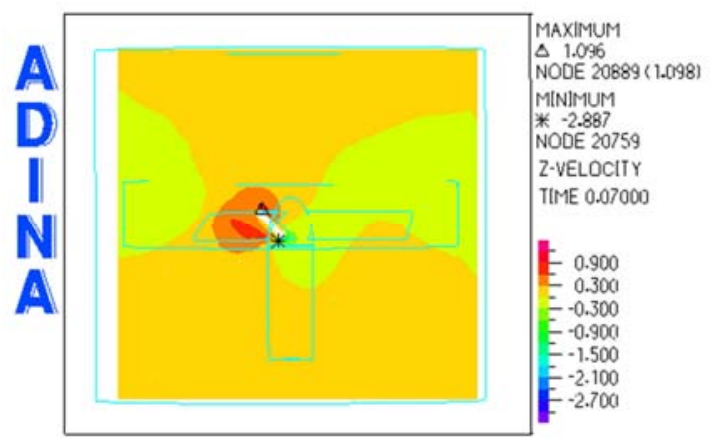

(a) The speed of section $\mathrm{X}$ under $0^{\circ}$

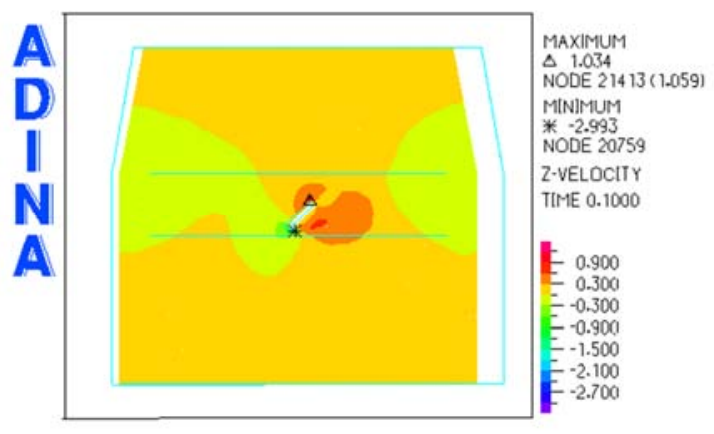

(b) The speed of section $\mathrm{X}$ under $10^{\circ}$

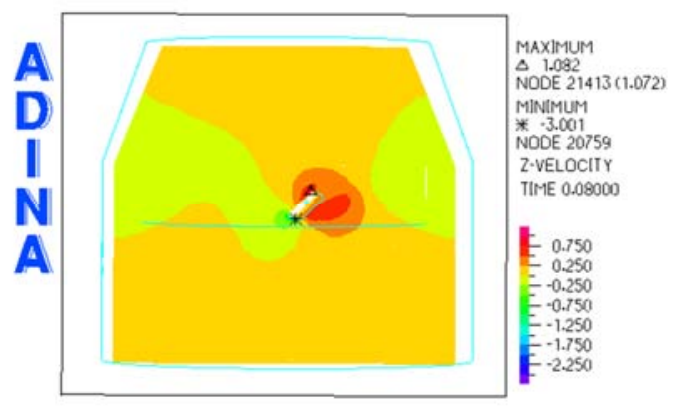

(c) The speed of section $\mathrm{X}$ under $20^{\circ}$

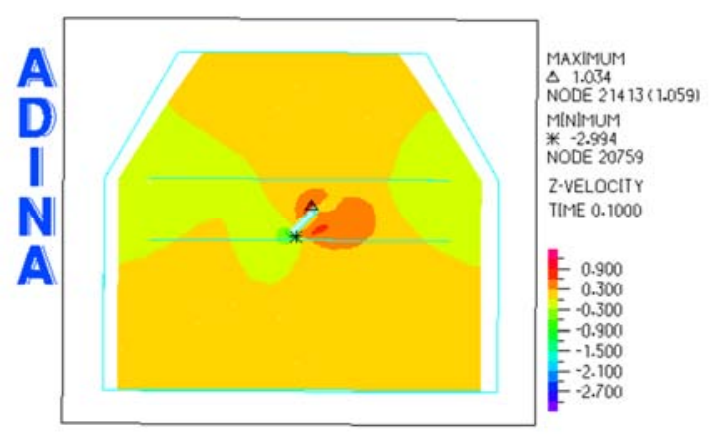

(d) The speed of section $\mathrm{X}$ under $30^{\circ}$

Fig. 4. The speed in direction $Z$ of flow field under different cone angle.

From Figure 4, we can see that the velocity distribution in the spray-duct. The velocity change in axis $\mathrm{Z}$ direction is shown in table 2 . From the table, when the cone angle is 0 degree the speed in the direction of axis $\mathrm{Z}$ is maximum, but the pressure angle is too small to ensure the effective transmission of fog particles. However, when the cone angle is 20 degrees, the speed in axis $Z$ has reached the maximum which is propitious to the effective transmission of fog particles.

Table 2. The speed in direction $Z$ under different cone angle.

\begin{tabular}{lll}
\hline \multirow{2}{*}{ cone angle } & \multicolumn{2}{l}{ Speed of X section in direction $\mathbf{Z}(\mathbf{m} / \mathbf{s})$} \\
\cline { 2 - 3 } & MAX & MIN \\
\hline $0^{\circ}$ & 1.096 & -2.887 \\
$10^{\circ}$ & 1.034 & -2.993 \\
$20^{\circ}$ & 1.082 & -3.001 \\
$30^{\circ}$ & 1.034 & -2.994 \\
\hline
\end{tabular}

\subsection{The analysis of Stress and Strain of the Fan}

The internal flow field characteristic parameter of the fan is closely related to the cone angle, and the liquid atomizing mainly is happened in the fan blade segment and guiding cone section. The change of guiding cone angle naturally leads to the changes in its internal flow field, and influences the effective range and floating effect of spray liquid after sprayed.

1) The influence of cone angle on stress of blades

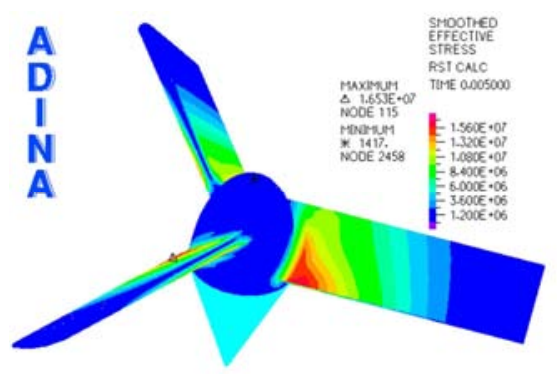

(a) The stress nephogram under $0^{\circ}$

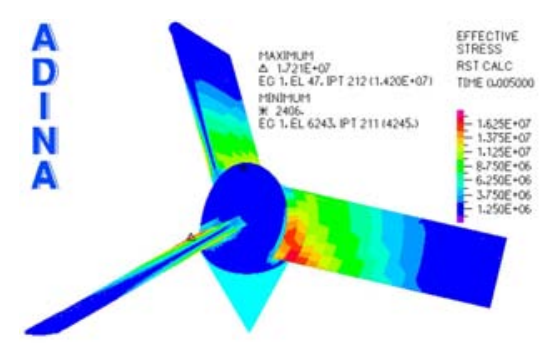

(b) The stress nephogram under $10^{\circ}$

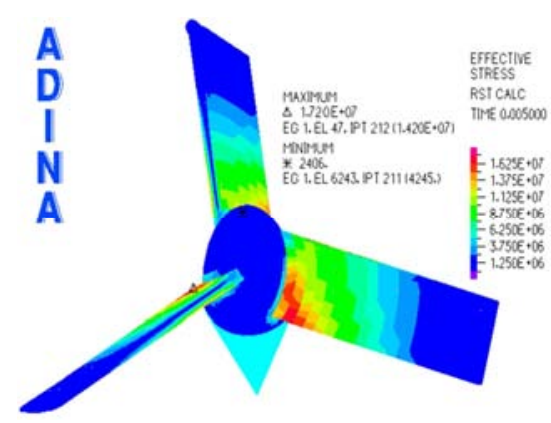

(c) The stress nephogram under $20^{\circ}$ 


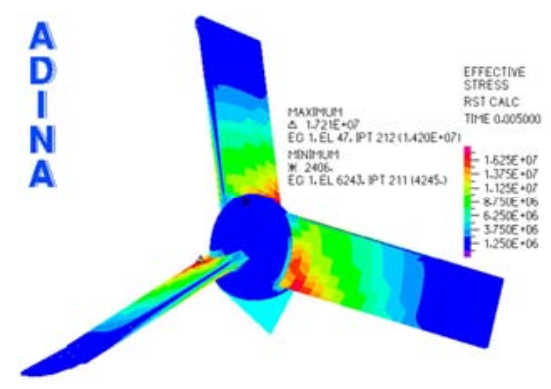

(d) The stress nephogram under $30^{\circ}$.

Fig. 5. The stress nephogram under different cone angle.

From Fig. 5, it can be seen that the fan blade roots bear alternate load, and the maximum and minimum pressure distribution in the blade roots. In order to observe the stress distribution of the fan blade under different guiding cone angle, the maximum external pressure and the maximum internal pressure are shown in Table 3.

Table 3. The max stress of structure field under different cone angle.

\begin{tabular}{lll}
\hline \multirow{2}{*}{ cone angle } & stress(MPa) & \\
\cline { 2 - 3 } & Exterior stress & Interior stress \\
\hline $0^{\circ}$ & 16.53 & 14.85 \\
$10^{\circ}$ & 17.21 & 15.93 \\
$20^{\circ}$ & 17.2 & 15.93 \\
$30^{\circ}$ & 17.21 & 15.93 \\
\hline
\end{tabular}

From table 3, when the cone angle increases, the fan blade stress increases, but a tiny drop when the cone angle is 20 degrees. It means that the load that fan blade bearing has a tiny drop at this time, which can guarantee the service life of the fan.

2 ) The influence of cone angle on strain of blades

From Figure 6, it can be seen that the deformation of the blades is mainly concentrated in the root of the blades. In order to observe the change of the strain of blades in detail, the maximum and the minimum strain value of the blades are shown in Table 4.

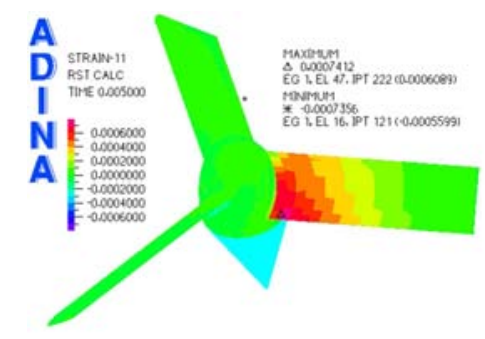

(a) The strain nephogram under $0^{\circ}$.

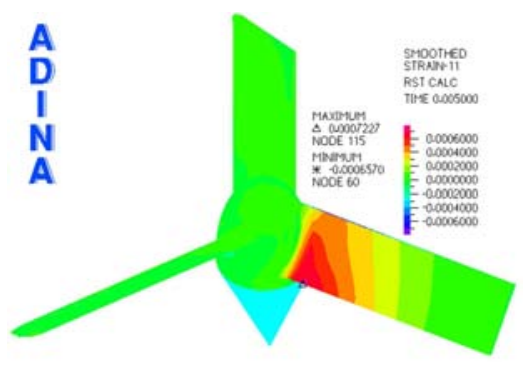

(b) The strain nephogram under $10^{\circ}$

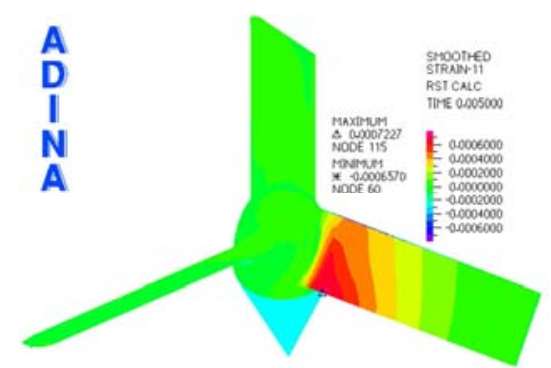

(c) The strain nephogram under $20^{\circ}$

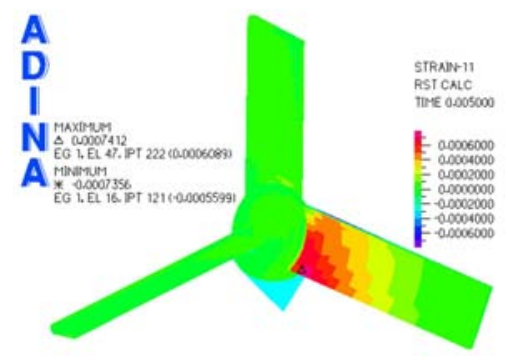

(d) The strain nephogram under $30^{\circ}$

Fig. 6. The strain nephogram under different cone angle.

Table 4. The strain of the fan under different cone angle.

\begin{tabular}{lllll}
\hline \multirow{2}{*}{$\begin{array}{l}\text { cone } \\
\text { angle }\end{array}$} & \multicolumn{2}{l}{ Exterior strain(x10-4m) } & \multicolumn{2}{l}{ Interior stress(x10-4m) } \\
\cline { 2 - 5 } & MAX & MIN & MAX & MIN \\
\hline $0^{\circ}$ & 7.227 & -6.57 & 6.156 & -6.172 \\
$10^{\circ}$ & 7.412 & -7.356 & 5.935 & -7.342 \\
$20^{\circ}$ & 7.227 & -6.57 & 6.765 & -5.19 \\
$30^{\circ}$ & 7.412 & -7.356 & 6.517 & -6.871 \\
\hline
\end{tabular}

From table 4 , it can be seen that when the minimum deformation of blades is 0 degrees and 20 degrees. The flow field in the 0 degree pressure angle and the velocity of the $Z$ direction are smaller, but the pressure angle of the 20 degree angle is appropriate. Therefore when the degree angle is 20 degree, the variation of the strain is the minimum, the alternate load of the blades is the minimum, the number of alternating load on the fan will increase, which can ensure the fatigue strength of the blades.

\section{Conclusion}

In the case of the guiding cone angle changes, simulated the influence of the spray flow field on the fan blade, and did the numerical analyzation. The conclusions are as follow:

(1) With the increase of the cone angle of the vehicle mounted sprayer, the pressure and velocity in the direction of the axis $\mathrm{Z}$ of the flow field also increased. The increasing pressure and speed can ensure the effective transmission of the fog particles, but also easily lead to the stress concentration in the root of the blade.

(2) Changing cone angle can effectively improve the performance of the spray fan, especially when the angle is 20 degrees, the internal pressure and the speed in $\mathrm{Z}$ direction of the spray fan can reach a relatively large value. but stress and strain of the blades reached the relatively small.

(3) In this paper, the contrastive analysis of simulation 
results and the experimental results are not carried out, and it will be added in the follow-up work in order to enhance the feasibility of the theory.

\section{References}

[1] Yang Chun, Li Qiushi, Yuan Wei, et al. Numerical and experimental investigation of the fan structure effects on performance. [J]. Journal of Aerospace Power, 2005, 20(3): 512 517. (in China)

[2] Lu Xiaolan, Fu Ximin, Wu Ping, et al. Influence of spray operating parameters on droplet deposition[J].Transactions of the Chinese Society for Agricultural Machinery, 2011, 42( 6): 70 75. ( in Chinese)

[3] Guo Feng, Chen Jiandong, Guo Hui, et al. Performance optimization and advance of $3 \mathrm{WF}-8$ air-assisted orchard sprayer [J].Journal of Agricultural Mechanization Research, 2010,32( 11): 48 56.( in Chinese)

[4] Song Shuran, Xia Houbing, Liu Hongshan, et al. Numerical simulation and experiment of structural optimization for air-blast sprayer[J]. Transactions of the Chinese Society for Agricultural Machinery, 2013, 44(6): 73 78. ( in Chinese)

[5] Liu Qing, Fu Zetian, Qi Lijun, et al. Characteristics optimization experiments of 9WZCD-25 air-blast and ultra low volume sprayer[J].Transactions of the Chinese Society for Agricultural Machinery, 2005, 36( 9): 44 47. ( in Chinese)

[6] Delele M A, Jaeken P, Debaer C, et al. CFD prototyping of an air-assisted orchard sprayer aimed at drift reduction [J]. Computers and Electronics in Agriculture, 2007, 55(1): 16 27.

[7] Endalew A M, Debaer C, Rutten N, et al. A new integrated CFD modeling approach towards air-assisted orchard spraying. Part I. Model development and effect of wind speed and direction on sprayer airflow [J]. Computers and Electronics in Agriculture, 2010, 71(2): 128 136.

[8] Tsay J R, Liang L S, Lu L H. Evaluation of an air-assisted boom spraying system under a no-canopy condition using CFD simulation [J]. Transactions of the ASAE, 2004, 47(6): $1887 \sim 1897$.

[9] Zhu H, Brazee R D, Derksen R C, et al. A specially designed air-assisted sprayer to improve spray penetration and air jet velocity distribution inside dense nursery crops $[\mathrm{J}]$. Transactions of the ASABE, 2006, 49(5): 1285 1294.

[10] Xing Jingtang, Zhou Sheng, Cui Erjie, et al. A survey on the fluid-solid interaction mechanics. [J]. Advances in Mechanics, 1997, 27(1): 19-38. ( in Chinese)

[11] Shang Jingtai. Manual for ventilator. [M]. Beijing: China Machine Press, 1996. ( in Chinese)

[12] Qiu Baijing, Yan Run, Ma Jing, et al. Research Progress Analysis of Variable Rate Sprayer Technology [J]. Transactions of the Chinese Society for Agricultural Machinery, 2015, 46(3): 59-72.

[13] Travis J. Esau, Qamar U. Zaman, Young K. et al. Spot-application of fungicide for wild blueberry using an automated prototype variable rate sprayer [J]. Precision Agriculture. 2014 (2) 